\title{
The Risk Factors of Early and Late-Onset Adjacent Segment Degeneration after Thoracolumbar Fusion Surgery
}

\author{
Dong-Whee Kim, Jung-Ho Kim, Dal-Sung Ryu, Seung-Hwan Yoon \\ Department of Neurosurgery, Inha University Hospital, Inha University College of Medicine, Incheon, Republic of Korea
}

Corresponding author: Seung-Hwan Yoon

Department of Neurosurgery, Inha University Hospital, Inha

University College of Medicine, 27, Inhang-ro, Jung-gu, Incheon 22332, Republic of Korea

Tel: $+82-32-890-2370$

Fax: +82-32-890-2374

E-mail: nsyoon@gmail.com

Received: August 10, 2020

Revised: September 17, 2020

Accepted: September 18, 2020
Objective: Adjacent segment degeneration (ASD) is a long-term complication that can occur after thoracolumbar fusion surgery. This study was undertaken to determine the risk factors of earlyonset ASD. Methods: We retrospectively reviewed patients who underwent thoracolumbar fusion at our institute for lumbar disease from January 2008 to December 2018. Potential risk factors examined included radiologic factors such as postoperative sagittal alignment, preexisting disc degeneration, and facet degeneration. For the analysis, patients were dichotomized based on times to ASD, that is, $<1$ year (the early group) and $\geq 1$ year (the late group). Results: During the 11-year study period, 693 patients underwent thoracolumbar fusion, and 92 (13.28\%) patients developed ASD; 34 (4.9\%) during the first postoperative year and 58 (8.4\%) at more than 1 year after surgery. The proportion of patients with a longer fusion length was greater in the early group $(p=0.0045)$. Postoperative lumbar lordosis ( $L L ; p=0.0007)$ and sagittal vertical axis (SVA) $(p=0.009)$ values were significantly different in the 2 groups, sacral slope (SS; $p<0.05)$ was smaller in the early group, and pelvic tilt (PT; $p=0.03$ ) was larger in the early group. However, pelvic incidence ( $P I ; p=0.72$ ) was no different in the 2 groups. Conclusion: The risk factors of early-onset ASD after thoracolumbar fusion surgery were confirmed to be the degree of fusion length and LL after surgery. SVA was found to possibly contribute to early-onset ASD. Although PI was similar in the 2 study groups, SS and PT were significantly different. So when you perform spinal fusion surgery, you should minimize fusion length and consider sagittal alignment.

Key Words: Intervertebral disc degeneration; Lumbar vertebrae; Risk factors; Spinal fusion

\section{INTRODUCTION}

Spinal fusion surgery is required for diverse degenerative lumbar diseases, such as spondylolisthesis, instability, herniated nucleus pulposus, stenosis, hypertrophic facet arthritis, and kyphosis due to vertebral compression fracture ${ }^{6,10,18,22)}$. Adjacent segment disease is a representative long-term complication after spinal fusion surgery and is defined as symptomatic degeneration that necessitates second surgery at a level above or below the index fusion level and adversely affects clinical outcomes $^{39)}$. In contrast, radiographic adjacent segment disease or adjacent segment degeneration (ASD) is defined as a pathological process at a disc adjacent to a prior fusion level and includes disc degeneration, segmental instability, or canal stenosis regardless of the presence of clinical symptoms ${ }^{15)}$.

ASD was first reported about 50 years ago ${ }^{19-21,24,37)}$. The recent dramatic increase in the number of spinal fusions has led to a corresponding increase in the incidence of ASD. The etiology of ASD is known to be multifactorial. Potential risk factors of ASD include posterior lumbar interbody fusion (PLIF), injury to the facet joint of an adjacent segment, fusion length, the presence of a preexisting degenerated disc, lumbar stenosis, age, osteoporosis, female gender, and a postmenopausal state $^{1,4,14,23,28,31)}$. Although many authors have evaluated the risk factors of ASD, clearly defined results lack due to study heterogeneities $^{11,12)}$.

Sagittal balance is closely associated with health-related quality of life, and thus, maintaining sagittal balance with adequate lumbar lordosis (LL) is an important goal of thoracolumbar fusion surgery. However, little is known about how sagittal imbalance affects the onset of ASD. To the best of our knowledge, no study has yet identified the risk factors of early and late-onset ASD. The present study was conducted to determine the incidence of ASD at a single institution over 11 years and to identify the clinical and radiologic risk factors of early and late-onset ASD with a focus on how sagittal imbalance affects ASD onset. To minimize confusion, we restricted enrolment to patients with radiologically confirmed ASD. 


\section{MATERIALS AND METHODS}

\section{Study Design}

This study was approved by our Institutional Review Board (IRB; IRB approval no. 2020-03-027). We analyzed all consecutive patients that underwent thoracolumbar fusion at our institution from January 2008 to December 2018. All medical records were reviewed retrospectively for clinical and radiologic data.

The following inclusion criteria were applied; the receipt of spinal fusion of the thoracolumbar spine due to degeneration or trauma, minimum age at surgery of 18 years, and minimum 18 months of follow-up. We excluded patients with tumorous, infectious conditions, a history of prior lumbar spine surgery, and concomitant scoliosis.

The early-onset group was defined as the detection of ASD at $<1$ year after initial surgery and the late-onset group as detection at $\geq 1$ year after surgery.

The radiographic variable and ASD data were collected from electronic medical records and operative reports. Follow-up radiologic test results of all patients were reviewed. We defined ASD as follows: In comparison with preoperative lateral flexion radiographs, conditions in which narrowing of disc height by $>3 \mathrm{~mm}$, posterior opening $>5^{\circ}$, and progress of slippage $>3 \mathrm{~mm}$. Magnetic resonance imaging (MRI) was also used to evaluate postoperative progression of disc degeneration and facet degeneration. In accordance with the definition of ASD, patients with radiologic changes and associated symptoms were investigated. Patient demographics and baseline characteristics were recorded for all subjects and included age, gender, days from initial operation to radiologic confirmation of ASD. Fusion was performed in the majority by posterolateral lumbar fusion using various types of interbody fusion techniques such as PLIF, transforaminal lumbar interbody fusion, and others. Surgical techniques used for fusion were determined by the surgeon's preference.
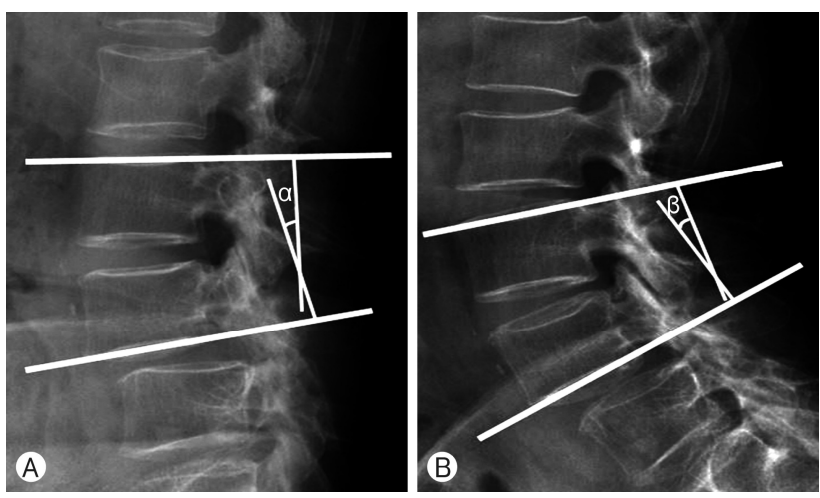

Fig. 1. Preoperative adjacent segmental flexion (A), extension (B) Cobb's angle $\alpha, \beta$. Range of motion $=\alpha-\beta$.

\section{Radiologic Evaluations}

Preoperative lateral dynamic radiographs were used to measure Cobb's angles of adjacent segments during flexion and extension (Fig. 1). Range of motion (ROM) was defined as the difference between these angles ${ }^{4}$. Preoperative MRI was used to investigate preexisting disc and facet degeneration at adjacent segments at the time of initial surgery. Disc degeneration on MRI was rated from grades 1 to 5 using the Pfirmann classification system ${ }^{26}$, and facet degeneration was rated using MR images from grade 0 to grade 3 using Weishaupt's classification ${ }^{36}$.

Sagittal alignment parameters measured on lateral radiographs were as follows: LL, pelvic incidence (PI), sacral slope (SS), pelvic tilt (PT), difference between PI and LL (PI-LL), and sagittal vertical axis (SVA; Fig. 2). These radiologic parameters were measured after operations to evaluate the effect of fusion surgery on ASD.

\section{Statistical Analysis}

Student $t$-tests were used to determine the significances of intergroup differences when variables were normally distributed. The incidence of ASD was analyzed. Potential risk factors were evaluated for univariate associations with ASD using the independent-samples $t$-test for continuous variables and the $\mathrm{X}^{2}$ test for categorical variables. The statistical analysis was performed using R version 3.6.3 (The R Foundation for Statistical Computing, Vienna, Austria). Results are presented as mean

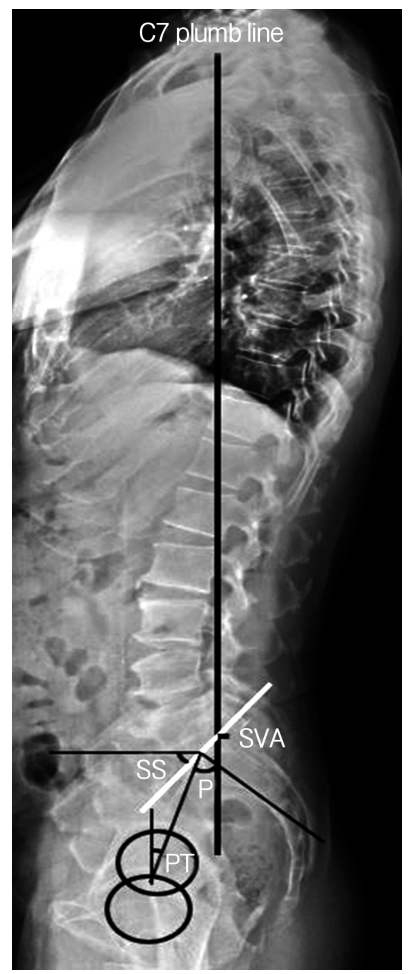

Fig. 2. The sagittal alignment parameters and pelvic parameters were obtained using standing lateral radiographs. The picture shows measurements of pelvic incidence $(\mathrm{PI})$, sacral slope (SS), pelvic tilt $(P T)$, sagittal vertical axis (SVA), and lumbar lordosis (LL). 
values \pm standard deviations or medians and interquartile ranges. Statistical significance was accepted for $\mathrm{p}$-values less than 0.05 .

\section{RESULTS}

During the 11-year study period, 1,121 patients underwent thoracolumbar fusion surgery. The 428 of these patients were excluded due to tumor, infection, prior spine surgery, adjacent segment decompression, or concomitant scoliosis. During the follow-up period, 92 patients developed ASD; an incidence of $13.28 \%$ (92/693). Of these 92 patients, ASD occurred at $<1$ year after surgery in 34 patients (4.9\%) and at $\geq 1$ year after

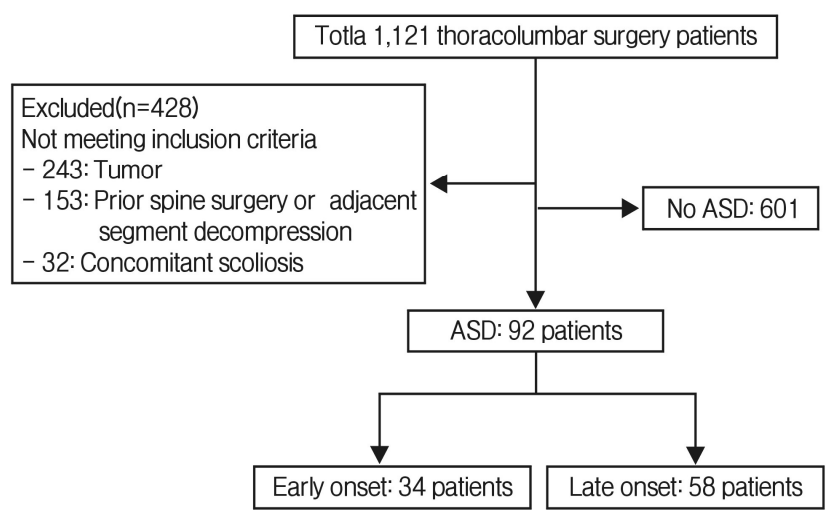

Fig. 3. Flowchart depicting patient selection. ASD: adjacent segment degeneration. surgery in $58(8.4 \%)$, and these patients constituted the 2 study groups, that is, the early and late-onset groups, respectively (Fig. 3).

Demographic characteristics (sex, age, and bone mineral density) were not significantly different in the 2 groups ( $p>0.05$ ) Patients in the early and late groups were diagnosed with ASD at $200.35 \pm 94.60$ and $1,323.31 \pm 466.76$ days postoperatively, respectively, and this difference was statistically significant $(\mathrm{p}<0.005)$. Fusion length was significantly greater in the early group ( $2.38 \pm 1.18$ vs. $1.74 \pm 0.9, \mathrm{p}=0.0045)$. Distributions of fusion lengths also differed significantly in the 2 groups $(\mathrm{p}=0.011$ ). However, types of fusion techniques used were not associated with time to ASD onset ( $\mathrm{p}=0.991$ ) (Table 1).

Pfirrmann and facet degeneration grades at adjacent segments were determined using preoperative MR images. However, no significant intergroup difference was found $(\mathrm{p}=0.09$, $\mathrm{p}=0.09$ respectively). Accordingly, preoperative degenerative changes were not correlated with time to ASD onset.

Sagittal alignment and pelvic parameters were measured in the 92 study subjects. Postoperative whole spine lateral X-rays showed SVA in the early group was $50.14 \pm 46.34$, which is slightly higher than normal, but that SVA in the late group was $26.76 \pm 36.53$, which is within the normal range, and these values were significantly different $(\mathrm{p}=0.009)$. LL measured after fusion surgery was $28.67 \pm 14.46^{\circ}$ in the early group and $37.83 \pm$ $10.57^{\circ}$ in the late group. Both angles were inadequate for normal lumbar lordosis, and the early group had a significantly smaller lumbar lordotic angle than the late group $(\mathrm{p} \leq 0.005)$. The SS in the early onset group was significantly lower than in the late-onset group $(20.76 \pm 7.86$ vs. $30.57 \pm 8.39, \mathrm{p}<0.005)$,

Table 1. Demographic characteristics of the early onset and late onset groups

\begin{tabular}{|c|c|c|c|}
\hline & Early onset $(n=34)$ & Late onset $(n=58)$ & $\mathrm{p}$-value \\
\hline Age (years) & $67.9 \pm 7.46$ & $65.9 \pm 7.98$ & 0.230 \\
\hline Sex & & & 0.130 \\
\hline Male & 9 (26.5\%) & $8(13.8 \%)$ & \\
\hline Female & 25 (73.5\%) & 50 (86.2\%) & \\
\hline \multicolumn{4}{|l|}{$\mathrm{BMD}$} \\
\hline Femur & $-1.84 \pm 1.05$ & $-1.61 \pm 1.13$ & 0.320 \\
\hline Lumbar & $-1.84 \pm 1.34$ & $-1.38 \pm 1.50$ & 0.130 \\
\hline Fusion length & $2.38 \pm 1.18$ & $1.74 \pm 0.9$ & $0.004^{*}$ \\
\hline 1 segment & 7 (20.6\%) & $28(48.3 \%)$ & $0.011^{*}$ \\
\hline 2 segments & $14(41.2 \%)$ & 21 (36.2\%) & \\
\hline$\geq 3$ segments & 13 (38.2\%) & $9(15.5 \%)$ & \\
\hline Types of fusion & & & 0.991 \\
\hline Only PLF & 27 (79.4\%) & $46(79.3 \%)$ & \\
\hline Interbody fusion & $7(20.6 \%)$ & $12(20.7 \%)$ & \\
\hline
\end{tabular}

The data is presented as mean \pm standard deviation or number (\%). BMD: bone mineral density; PLF: posterolateral lumbar fusion. $p<0.05$. 
Table 2. Radiologic findings of the early and late onset groups

\begin{tabular}{|c|c|c|c|}
\hline & Early onset $(n=34)$ & Late onset $(n=58)$ & $\mathrm{p}$-value \\
\hline \multicolumn{4}{|l|}{ Preoperative } \\
\hline Pfirrmann grade & $3.09 \pm 0.97$ & $2.76 \pm 0.84$ & 0.090 \\
\hline Facet degeneration grade & $1.71 \pm 0.72$ & $1.47 \pm 0.62$ & 0.090 \\
\hline \multicolumn{4}{|l|}{ Segmental angle $\left(^{\circ}\right)$} \\
\hline Flexion & $6.95 \pm 5.67$ & $5.08 \pm 5.19$ & 0.090 \\
\hline Extension & $13.31 \pm 6.74$ & $13.11 \pm 6.86$ & 0.890 \\
\hline Range of motion & $20.26 \pm 11.59$ & $18.19 \pm 10.85$ & 0.370 \\
\hline \multicolumn{4}{|l|}{ Postoperative } \\
\hline Sagittal vertical axis $(\mathrm{mm})$ & $50.14 \pm 46.34$ & $26.76 \pm 36.53$ & $0.009^{*}$ \\
\hline Lumbar lordosis $\left({ }^{\circ}\right)$ & $28.67 \pm 14.46$ & $37.83 \pm 10.57$ & $0.001^{*}$ \\
\hline \multicolumn{4}{|l|}{ Pelvic parameter $\left({ }^{\circ}\right)$} \\
\hline Pelvic incidence & $49.06 \pm 11.7$ & $53.76 \pm 12.15$ & 0.720 \\
\hline Sacral slope & $20.76 \pm 7.86$ & $30.57 \pm 8.39$ & $<0.005^{*}$ \\
\hline Pelvic tilt & $28.31 \pm 11.94$ & $23.19 \pm 10.36$ & $0.030^{*}$ \\
\hline Pelvic incidence-lumbar lordosis mismatch $\left(^{\circ}\right)$ & $20.39 \pm 13.65$ & $15.94 \pm 14.7$ & 0.190 \\
\hline
\end{tabular}

The data is presented as mean \pm standard deviation.

${ }^{*} \mathrm{p}<0.05$.

Table 3. Results of multivariate logistic regression analysis regarding early onset adjacent segment degeneration

\begin{tabular}{ccc}
\hline \hline \multirow{2}{*}{ Risk factors } & \multicolumn{2}{c}{ Logistic regression } \\
\cline { 2 - 3 } & $\mathrm{p}$-value & $\mathrm{OR}(95 \% \mathrm{Cl})$ \\
\hline Fusion length & 0.039 & $1.61(1.02-2.51)$ \\
LL & 0.008 & $0.95(0.91-0.99)$ \\
\hline
\end{tabular}

OR: odds ratio; $\mathrm{Cl}$ : confidence interval; LL: lumbar lordosis.

but PT in the early group was greater than in the late group. (28.31 \pm 11.94 vs. $23.19 \pm 10.36, \mathrm{p}=0.03)$ However, other pelvic parameters including PI and PI minus LL mismatch did not reveal a significant difference between the 2 groups (Table 2).

Using the clinically meaningful risk factors, a multivariate logistic regression model was constructed (Table 3 ). The factor with the highest odds ratio (OR) was fusion length (OR, 1.61; $95 \%$ confidence interval [CI], 1.02-2.51). LL showed a negative correlation (OR, 0.95; 95\% CI, 0.91-0.99)

\section{DISCUSSION}

Fusion is a well-established surgical technique for managing many types of spinal disease. Recent advances in medical devices and biologics have substantially increased the number of fusion surgeries performed worldwide. However, fusion itself causes tremendous stress to facet joints and discs of adjacent segments ${ }^{16)}$, increases mechanical stress at adjacent segments, and irreversibly changes spinal biomechanics ${ }^{2)}$.

Radiographic degeneration at adjacent segments is a com- mon finding but does not necessarily correlate with clinical symptoms. According to related reports, the incidence of asymptomatic radiographic ASD ranges from as little as $8 \%$ to $100 \%$, whereas that of reported symptomatic ASD ranges from 5.2\% to $18.5 \%^{13,25,27)}$, and only a small proportion of these cases require a second surgery ${ }^{7)}$. In the present study, ASD was defined as the presence of radiologic change at adjacent segments regardless of symptoms, and we found that the overall incidence of ASD was $13.28 \%$ and that 34 cases (4.9\%) occurred at $<1$ year and 58 cases (8.4\%) at $\geq 1$ year. Other studies have reported similar findings. Wang et al. ${ }^{34)}$ reported a $6.3 \%$ incidence of postoperative ASD in 15 patients with degenerative lumbar disorders, which included lumbar disc herniation, lumbar spinal stenosis, degenerative lumbar spondylolisthesis.

This study shows that a greater fusion length was strongly associated with early ASD (OR, 1.61; 95\% CI, 1.02-2.51), concurs with previous reports. Song et $\mathrm{al}^{32)}$ reported that the prevalence of ASD is high when the fusion range is above 3 segments. Because stress at adjacent segment increases in proportion with prior fusion length, remnant mobile segments are easily damaged. Therefore, spine surgeons always need to bear in mind the possibility of ASD and consequent fusion extension. Fusion should be decided not only by considerations of current pathology but also of overall spinal column structure, and the proper fusion site should be determined based on careful medical history-taking and physical and radiologic evaluations ${ }^{30)}$.

In the current study, degenerative alteration of intervertebral discs and facet joints of adjacent segments before surgery was not associated with time to ASD onset. It remains controversial whether degenerative changes of adjacent segment interver- 
tebral discs and facet joints affect the progression of ASD. Gillet $^{8)}$ reported that segments with degenerative changes before surgery did not play a significant role in terms of long-term results, and thus, performed fusion surgery only on segments related to current symptoms. On the contrary, there have been reports that degenerative changes in adjacent segments after surgery were accelerated after initial fusion and increased the prevalence of adjacent segment disease $e^{3,6,9,35)}$.

We measured preoperative segmental angles of upper and lower lumbar vertebrae adjacent to segments at which ASD occurred after surgery. The segmental angle was determined by obtaining the flexion, extension, and ROM, and there was no intergroup difference observed ${ }^{38)}$. Initially, we believed that preoperative instability might cause ASD during the early postoperative period, but no clear association was observed.

PT importantly influences spinal alignment and ASD. According to a study conducted by Di Martino et al..$^{5}$, patients with a PT $>20^{\circ}$ and an SS $\angle 40^{\circ}$ represent a high-risk group for clinically evident ASD and subsequent reintervention. Pelvic retroversion and hypolordosis are the 2 main mechanisms of compensation that create an unbalanced spine ${ }^{5)}$. Yamasaki et al. ${ }^{37)}$ found greater PT results in reduced LL, hip and knee flexion contracture, and nerve or muscle disorders. In our study, postoperative PT and SS values in the early and late ASD groups were significantly different. Therefore, we conclude lower SS and higher PT are risk factors of early-onset ASD.

It has been consistently suggested that changes in sagittal plane anatomy are positively associated with the incidence of $\mathrm{ASD}^{17,29)}$. Kumar et al. ${ }^{14)}$ showed more significant degenerative changes at adjacent levels occurred when $\mathrm{C} 7$ plumb line alignment was abnormal after lumbar fusion surgery. In addition, many studies have concluded that the incidence of ASD is lower when the postoperative lumbar lordotic angle remains in the normal range. The present study confirmed that ASD occurs rapidly when the degree of sagittal imbalance is severe. When this plumb line is antepulsed, it means that pelvic compensation either did not occur (e.g., hip arthritis) or was insufficient to correct a grossly abnormal amount of antepulsion. The instantaneous axis of rotation of a structurally normal spine passes through the anterior third of lumbar disc spaces and the moment arm of the center of mass is balanced by the moment arm of spinal muscles. However, in the presence of antepulsion, the moment arm of the center of mass increases and increases loading of unfused motion segments. Another reason for antepulsion-associated disc degeneration concerns extensor muscle activity during attempts to maintain balance ${ }^{33)}$. It has been shown that compressive loading of discs is highest during trunk extension exercises ${ }^{1)}$.

The present study has several limitations. First, because of its cross-sectional design, the associations found between risk factors and ASD do not imply cause-and-effect relationships. Second, we compared differences between early and late-onset ASD groups, and had a group that did not develop ASD been added, more meaningful results might have been obtained. Third, due to the relatively low incidence of ASD, limited numbers of patients were enrolled. We suggest a multi-center study be conducted on symptomatic ASD.

\section{CONCLUSION}

The incidence of ASD after thoracolumbar fusion was found to be $13.28 \%$. Early-onset ASD was associated with greater fusion length, inadequate acquisition of lumbar lordosis, lower SS, and higher PT. Therefore, these findings indicate that to reduce the occurrence of ASD, fusion length be minimized by integrating only necessary parts as determined by accurate physical examination, and that sagittal alignment be corrected as close to normal as possible.

\section{CONFLICTS OF INTEREST}

No potential conflict of interest relevant to this article was reported.

\section{REFERENCES}

1. Callaghan JP, Gunning JL, McGill SM: The relationship between lumbar spine load and muscle activity during extensor exercises. Phys Ther 78:8-18, 1998

2. Chen CS, Cheng CK, Liu CL, Lo WH: Stress analysis of the disc adjacent to interbody fusion in lumbar spine. Med Eng Phys 23:483-491, 2001

3. Cho KJ, Park SR, Moon KH, Lee KY, Kim KH, Kang SB: Progression of preoperative degeneration of the adjacent segment after instrumented lumbar arthrodesis. J Korean Orthop Assoc 42:453-460, 2007

4. Dekutoski MB, Schendel MJ, Ogilvie JW, Olsewski JM, Wallace LJ, Lewis JL: Comparison of in vivo and in vitro adjacent segment motion after lumbar fusion. Spine (Phila Pa 1976) 19: 1745-1751, 1994

5. Di Martino A, Quattrocchi CC, Scarciolla L, Papapietro N, Beomonte Zobel B, Denaro V: Estimating the risk for symptomatic adjacent segment degeneration after lumbar fusion: Analysis from a cohort of patients undergoing revision surgery. Eur Spine J 23 Suppl 6:693-698, 2014

6. Etebar S, Cahill DW: Risk factors for adjacent-segment failure following lumbar fixation with rigid instrumentation for degenerative instability. J Neurosurg 90:163-169, 1999

7. Ghiselli G, Wang JC, Bhatia NN, Hsu WK, Dawson EG: Adjacent segment degeneration in the lumbar spine. J Bone Joint Surg Am 86:1497-1503, 2004

8. Gillet P: The fate of the adjacent motion segments after lumbar fusion. J Spinal Disord Tech 16:338-345, 2003

9. Ha KY, Kim KW, Park SJ, Lee YH: Changes of the adjacentunfused mobile segment after instrumental lumbar fusion: More than 5-years follow-up. J Korean Soc Spine Surg 5:205-214, 1998 
10. Ha KY, Son JM, Im JH, Oh IS: Risk factors for adjacent segment degeneration after surgical correction of degenerative lumbar scoliosis. Indian J Orthop 47:346-351, 2013

11. Her Y, Kang SH, Cho YJ, Yang JS, Jeon JP, Choi HJ: Factors associated with longer postoperative outpatient follow-up duration in patients with single lumbar disc herniation: A noncomplicated patient cohort study. Neurospine 15:225-230, 2018

12. Ilves $\mathrm{OE}$, Neva $\mathrm{MH}$, Häkkinen $\mathrm{K}$, Dekker J, Kraemer WJ, Tarnanen S, et al.: Trunk muscle strength after lumbar spine fusion: A 12-month follow-up. Neurospine 16:332-338, 2019

13. Khanduja S, Loomba V, Salama-Hannah J, Upadhyay A, Khanduja N, Chauhan G: Retrospective review of magnetic resonance imaging of the lumbosacral spine: Are we overinvestigating? Neurospine 15:383-387, 2018

14. Kumar MN, Baklanov A, Chopin D: Correlation between sagittal plane changes and adjacent segment degeneration following lumbar spine fusion. Eur Spine J 10:314-319, 2001

15. Kuo CH, Huang WC, Wu JC, Tu TH, Fay LY, Wu CL, et al.: Radiological adjacent-segment degeneration in L4-5 spondylolisthesis: comparison between dynamic stabilization and minimally invasive transforaminal lumbar interbody fusion. J Neurosurg Spine 29:250-258, 2018

16. Lai PL, Chen LH, Niu CC, Fu TS, Chen WJ: Relation between laminectomy and development of adjacent segment instability after lumbar fusion with pedicle fixation. Spine (Phila Pa 1976) 29:2527-2532, 2004

17. Lazennec JY, Ramaré S, Arafati N, Laudet CG, Gorin M, Roger $\mathrm{B}$, et al.: Sagittal alignment in lumbosacral fusion: relations between radiological parameters and pain. Eur Spine J 9:47-55, 2000

18. Lee CS, Hwang CJ, Lee SW, Ahn YJ, Kim YT, Lee DH, et al.: Risk factors for adjacent segment disease after lumbar fusion. Eur Spine J 18:1637-1643, 2009

19. Lee JC, Kim Y, Soh JW, Shin BJ: Risk factors of adjacent segment disease requiring surgery after lumbar spinal fusion: comparison of posterior lumbar interbody fusion and posterolateral fusion. Spine (Phila Pa 1976) 39:E339-E345, 2014

20. Liang J, Dong Y, Zhao H: Risk factors for predicting symptomatic adjacent segment degeneration requiring surgery in patients after posterior lumbar fusion. J Orthop Surg Res 9:97, 2014

21. Malveaux WM, Sharan AD: Adjacent segment disease after lumbar spinal fusion: A systematic review of the current literature. Semin Spine Surg 23:266-274, 2011

22. Masevnin S, Ptashnikov D, Michaylov D, Meng H, Smekalenkov $\mathrm{O}$, Zaborovskii N: Risk factors for adjacent segment disease development after lumbar fusion. Asian Spine J 9:239-244, 2015

23. Matsumoto T, Okuda S, Maeno T, Yamashita T, Yamasaki R, Sugiura T, et al.: Spinopelvic sagittal imbalance as a risk factor for adjacent-segment disease after single-segment posterior lumbar interbody fusion. J Neurosurg Spine 26:435-440, 2017

24. Nakashima H, Kawakami N, Tsuji T, Ohara T, Suzuki Y, Saito $\mathrm{T}$, et al.: Adjacent segment disease after posterior lumbar interbody fusion: Based on cases with a minimum of 10 years of follow-up. Spine (Phila Pa 1976) 40:E831-E841, 2015
25. Park P, Garton HJ, Gala VC, Hoff JT, McGillicuddy JE: Adjacent segment disease after lumbar or lumbosacral fusion: review of the literature. Spine (Phila Pa 1976) 29:1938-1944, 2004

26. Pfirrmann CW, Metzdorf A, Zanetti M, Hodler J, Boos N: Magnetic resonance classification of lumbar intervertebral disc degeneration. Spine (Phila Pa 1976) 26:1873-1878, 2001

27. Rai RR, Shah Y, Shah S, Palliyil NS, Dalvie S: A radiological study of the association of facet joint tropism and facet angulation with degenerative spondylolisthesis. Neurospine 16:742747, 2019

28. Ren C, Song Y, Liu L, Xue Y: Adjacent segment degeneration and disease after lumbar fusion compared with motion-preserving procedures: a meta-analysis. Eur J Orthop Surg Traumatol 24 Suppl 1:S245-S253, 2014

29. Schlegel JD, Smith JA, Schleusener RL: Lumbar motion segment pathology adjacent to thoracolumbar, lumbar, and lumbosacral fusions. Spine (Phila Pa 1976) 21:970-981, 1996

30. Shin JJ: Comparison of adjacent segment degeneration, cervical alignment, and clinical outcomes after one- and multilevel anterior cervical discectomy and fusion. Neurospine 16:589-600, 2019

31. Song KJ, Choi BW, Jeon TS, Lee KB, Chang H: Adjacent segment degenerative disease: is it due to disease progression or a fusion-associated phenomenon? Comparison between segments adjacent to the fused and non-fused segments. Eur Spine J 20: 1940-1945, 2011

32. Song KJ, Ko JH, Jung YR: Analysis of risk factors of adjacent segment disease with lumbar and lumbosacral arthrodesis for the degenerative lumbar spinal disorders. J Korean Orthop Assoc 51:54-60, 2016

33. Swinkels A, Dolan P: Regional assessment of joint position sense in the spine. Spine (Phila Pa 1976) 23:590-597, 1998

34. Wang H, Ma L, Yang D, Wang T, Liu S, Yang S, et al.: Incidence and risk factors of adjacent segment disease following posterior decompression and instrumented fusion for degenerative lumbar disorders. Medicine (Baltimore) 96:e6032, 2017

35. Weinhoffer SL, Guyer RD, Herbert M, Griffith SL: Intradiscal pressure measurements above an instrumented fusion. A cadaveric study. Spine (Phila Pa 1976) 20:526-531, 1995

36. Weishaupt D, Zanetti M, Boos N, Hodler J: MR imaging and CT in osteoarthritis of the lumbar facet joints. Skeletal Radiol 28:215-219, 1999

37. Yamasaki K, Hoshino M, Omori K, Igarashi H, Nemoto Y, Tsuruta $\mathrm{T}$, et al.: Risk factors of adjacent segment disease after trans foraminal inter-body fusion for degenerative lumbar disease. Spine (Phila Pa 1976) 42:E86-E92, 2017

38. Yang KH, Kim NK, Kim YS, Ko Y, Oh SH, Oh SJ, et al.: Lumbar spinal instability and its radiologic findings. J Korean Neurosurg Soc 29:78-86, 2000

39. Zhong ZM, Deviren V, Tay B, Burch S, Berven SH: Adjacent segment disease after instrumented fusion for adult lumbar spondylolisthesis: Incidence and risk factors. Clin Neurol Neurosurg 156:29-34, 2017 